\title{
Optimizing Bacterial Cellulose Production Towards Materials for Water Remediation
}

Víctor Calvo ${ }^{1}$, Javier Torrubia ${ }^{2}$, Domingo Blanco², Enrique García-Bordeje ${ }^{1}$, Wolfgang K. Maser $^{1}$, Ana M. Benito ${ }^{1}$, Jose M. González-Domínguez ${ }^{1 \star}$

1) Group of Carbon Nanostructures and Nanotechnology, Instituto de Carboquímica ICBCSIC, C/Miguel Luesma Castán 4, 50018, Zaragoza, Spain

2) Department of Animal Production and Food Science, Veterinary Faculty, Instituto Agroalimentario de Aragón (IA2), C/ Miguel Servet 177, 50013 Zaragoza, Spain

Cellulose is a renewable alternative to mass consumption plastics, but its manufacture by the classical methods is not sustainable due to the use of large amounts of strong acids, bases and/ or organic species (e.g. ionic liquids) in its production, generating many residues. Bacterial cellulose (BC) has a simpler processing because it is much more cleanly generated. In this work, BC hydrogels, stemming from Komagateibacter xylinus bacteria, has been optimized in terms of bacterial culture and subsequently tailored in their physical properties after drying, giving rise to aerogels and xerogels. These are compared in order to ascertain how the bacterial culture conditions $(\mathrm{pH}$, carbon and nitrogen sources) and the raw hydrogels processing determine their thermal stability, crystallinity index, swelling ratio and flammability. The most notable results are the influence of the drying method on the swelling ratio and the carbon source on the thermal stability. It is possible to control the BC hydrogel properties by rationally selecting the appropriate drying method. In this regard, the aerogels (obtained by lyophilisation) have a much larger sorption capacity and a higher porosity, whereas xerogels (obtained by drying in open air) are more compact, as observed by SEM. Contrary to the aerogels, the BC xerogels are non-flammable. The $\mathrm{pH}$ of the culture medium does not have a great influence on the thermal stability of the xerogels and aerogels, but it is important for the hydrogel production rate and slightly influences the $\mathrm{Cl}$. The carbon source has a greater influence in the thermal stability of the final materials. Fructose provides a higher thermal stability; however, its dry materials are flammable; glucose and saccharose show an inverse trend as compared to fructose. The advantage of glucose over the fructose and saccharose is its somewhat faster production rate.

Finally, a feasible application of $\mathrm{BC}$ aerogels is presented by treating contaminated water and by capturing water within a nonpolar solvent, taking advantage of the dry BC sorption capacity. The accomplishment of a sorption test demonstrates that BC aerogels, once optimized in production and physical properties, can be applied as an efficient sorbent, taking advantage of their porosity and hydrophilic character. There are other possible implementations, such as filters to purify water, with critical implications in human safety and defence against chemical threats. 